PROCEEDINGS OF THE

AMERICAN MATHEMATICAL SOCIETY

Volume 131, Number 2, Pages 343-349

S 0002-9939(02)06553-X

Article electronically published on June 5, 2002

\title{
A NEW INVARIANT OF STABLE EQUIVALENCES OF MORITA TYPE
}

\author{
ZYGMUNT POGORZAEY
}

(Communicated by Martin Lorenz)

Dedicated to Professor Idun Reiten on the occasion of her sixtieth birthday

\begin{abstract}
It was proved in an earlier paper by the author that the Hochschild cohomology algebras of self-injective algebras are invariant under stable equivalences of Morita type. In this note we show that the orbit algebra of a selfinjective algebra $A$ (considered as an $A$ - $A$-bimodule) is also invariant under stable equivalences of Morita type, where the orbit algebra is the algebra of all stable $A$ - $A$-bimodule morphisms from the non-negative Auslander-Reiten translations of $A$ to $A$.
\end{abstract}

\section{INTRODUCTION}

Let $K$ be a fixed field. In representation theory of finite-dimensional associative $K$-algebras with identity elements, stable equivalences of Morita type seem to be of particular interest. They arose in representation theory of finite groups (see 44). Rickard proved that if two self-injective $K$-algebras are derived equivalent, then they are stably equivalent of Morita type [15]. Moreover, in [15] Rickard generalized a result of Happel [7] and proved that the Hochschild cohomology algebras of finite-dimensional $K$-algebras are invariant under derived equivalences. Rickard's result was generalized in [13, where it was proved that the Hochschild cohomology algebras of finite-dimensional self-injective $K$-algebras are also invariant under stable equivalences of Morita type.

Now suppose that $\mathcal{A}$ is an additive $K$-category. Then for any $K$-linear endofunctor $F: \mathcal{A} \rightarrow \mathcal{A}$ and any fixed object $X$ we define an algebra $\mathbb{A}(F ; X)$ of $F$ in $X$ as follows. The algebra $\mathbb{A}(F ; X)=\bigoplus_{n=0}^{\infty} \operatorname{Hom}_{\mathcal{A}}\left(F^{n}(X), X\right)$ as $K$-linear spaces and the multiplication of a homogeneous element $u: F^{n}(X) \rightarrow X$ of degree $n$ by a homogeneous element $v: F^{m}(X) \rightarrow X$ of degree $m$ is given by the composition $v u=\left[F^{m+n}(X) \stackrel{F^{n}(v)}{\longrightarrow} F^{n}(X) \stackrel{u}{\longrightarrow} X\right]$. Such algebras were studied by Lenzing in 10] and Kerner in 9. A particular example of such an algebra is the Hochschild cohomology algebra $H H(A)$ of a finite-dimensional self-injective $K$-algebra $A$. In fact $H H(A) \cong \mathbb{A}\left(\Omega_{A^{e}} ; A\right)$ where $A^{e}=A \otimes_{K} A^{o p}$ is the enveloping algebra of $A$ and $\Omega_{A^{e}}: \underline{\bmod }\left(A^{e}\right) \rightarrow \underline{\bmod }\left(A^{e}\right)$ is the Heller's loop-space functor on the stable category of the right finite-dimensional $A^{e}$-modules.

Received by the editors May 2, 2001 and, in revised form, September 6, 2001

2000 Mathematics Subject Classification. Primary 16D50; Secondary 16G20.

(C)2002 American Mathematical Society 
The main result of 13 shows that if $A$ is a symmetric $K$-algebra, then the algebra $\mathbb{A}\left(\tau_{A^{e}} ; A\right)$ is also invariant under stable equivalences of Morita type, where $\tau_{A^{e}}: \underline{\bmod }\left(A^{e}\right) \rightarrow \underline{\bmod }\left(A^{e}\right)$ is the Auslander-Reiten translation 3 . Our objective is to prove a similar result for stable equivalences of Morita type between arbitrary self-injective $K$-algebras.

The main result of this note is the following.

Theorem 1.1. Let $A, B$ be self-injective, finite-dimensional $K$-algebras. If $A$ and $B$ are stably equivalent of Morita type, then the algebras $\mathbb{A}\left(\tau_{A^{e}} ; A\right)$ and $\mathbb{A}\left(\tau_{B^{e}} ; B\right)$ are isomorphic.

As a consequence of this result we also obtain the following.

Theorem 1.2. Let $A, B$ be self-injective, finite-dimensional $K$-algebras which are derived equivalent. Then the algebras $\mathbb{A}\left(\tau_{A^{e}} ; A\right)$ and $\mathbb{A}\left(\tau_{B^{e}} ; B\right)$ are isomorphic.

A special case of a stable equivalence of Morita type is a Morita equivalence. In this case the above results are obvious.

\section{Preliminaries}

For a finite-dimensional $K$-algebra $A$ denote by $\bmod (A)$ the category of the finitedimensional right $A$-modules. The stable category $\underline{\bmod }(A)$ of $\bmod (A)$ (or shortly of $A$ ) modulo projectives is the quotient category $\bmod (A) / \mathcal{P}$, where $\mathcal{P}$ is the two-sided ideal in $\bmod (A)$ consisting of the morphisms which factorize through projective $A$ modules. For any two objects $X, Y$ of $\underline{\bmod }(A)$ we denote the morphism space $\operatorname{Hom}_{\underline{\bmod }(A)}(X, Y)$ by $\underline{\operatorname{Hom}}_{A}(X, Y)$. Every element of $\underline{\operatorname{Hom}}_{A}(X, Y)$ is a coset $\underline{f}$ of a morphism $f \in \operatorname{Hom}_{A}(X, Y)$ modulo $\mathcal{P}(X, Y)$.

The enveloping algebra $A^{e}$ of an algebra $A$ is the algebra $A \otimes_{K} A^{o p}$, where $A^{\text {op }}$ stands for the opposite algebra. We shall use $D$ for the usual duality. Thus $D=\operatorname{Hom}_{K}(-, K)$.

We have two self-equivalences on $\underline{\bmod }(A)$ in case $A$ is self-injective. One of them is Heller's loop-space functor $\Omega_{A}: \underline{\bmod }(A) \rightarrow \underline{\bmod }(A)$ [8]. The other one is the Auslander-Reiten translation $\tau_{A}: \underline{\bmod }(A) \rightarrow \underline{\bmod }(A)$ [3]. These two functors are related to one another by the following well-known formula [5]:

$$
\tau_{A} \cong \Omega_{A}^{2} \circ\left(-\otimes_{A} D(A)\right) .
$$

We shall frequently use the following properties. If two finite-dimensional $K$ algebras $A$ and $B$ are self-injective, then the tensor product algebra $A \otimes_{K} B^{o p}$ is also self-injective. In particular the enveloping algebra $A^{e}$ of a self-injective algebra $A$ is also self-injective. Every right, finite-dimensional $A \otimes_{K} B^{o p}$-module can be interpreted as a finite-dimensional $B$ - $A$-bimodule with a central action of $K$, and conversely, every such bimodule can be considered as a right $A \otimes_{K} B^{o p}$-module. Then we shall not distinguish between right $A \otimes_{K} B^{o p}$-modules and $B$ - $A$-bimodules.

A finite-dimensional $B$-A-bimodule $X$ is said to be left-right projective if it is projective as a left $B$-module and as a right $A$-module. In general there is a lot of indecomposable $B$ - $A$-bimodules which are left-right projective (see [14]). The $A$ - $A$-bimodule $A$ is a good example of a left-right projective $A$ - $A$-bimodule which is not a projective one.

Lemma 2.1. Let $A$ be a finite-dimensional, self-injective $K$-algebra. Then $\tau_{A^{e}}(A)$ $\cong A \otimes_{A^{e}} D\left(A^{e}\right) \otimes_{A} \Omega_{A^{e}}^{2}(A)$ in $\underline{\bmod }\left(A^{e}\right)$. 
Proof. We infer from $\tau_{A^{e}} \cong \Omega_{A^{e}}^{2} \circ\left(-\otimes_{A^{e}} D\left(A^{e}\right)\right)$ that $\tau_{A^{e}}(A) \cong \Omega_{A^{e}}^{2}\left(A \otimes_{A^{e}} D\left(A^{e}\right)\right)$. But we know from [11] that for any left-right projective $A$ - $A$-bimodule $X$ it holds that $\Omega_{A^{e}}^{2}(X) \cong X \otimes_{A} \Omega_{A^{e}}^{2}(A)$ in $\underline{\bmod }\left(A^{e}\right)$. Thus $\tau_{A^{e}}(A) \cong A \otimes_{A^{e}} D\left(A^{e}\right) \otimes_{A} \Omega_{A^{e}}^{2}(A)$ in $\underline{\bmod }\left(A^{e}\right)$ which finishes our proof.

We also have the following dual version of the above lemma.

Lemma 2.2. Let $A$ be a finite-dimensional, self-injective $K$-algebra. Then $\tau_{A^{e}}^{-1}(A)$ $\cong A \otimes_{A^{e}} D\left(A^{e}\right) \otimes_{A} \Omega_{A^{e}}^{-2}(A)$ in $\underline{\bmod }\left(A^{e}\right)$.

Proof. Use dual arguments to those in the proof of Lemma 2.1.

Lemma 2.3. Let $A$ be a finite-dimensional, self-injective $K$-algebra. Then for every indecomposable, left-right projective $A$-A-bimodule $X$ which is not projective there is an isomorphism $\tau_{A^{e}}(X) \cong X \otimes_{A} \tau_{A^{e}}(A)$ in $\underline{\bmod }\left(A^{e}\right)$.

Proof. We infer by the above formula that $\tau_{A^{e}}(X) \cong \Omega_{A^{e}}^{2}\left(X \otimes_{A^{e}} D\left(A^{e}\right)\right)$. But $X \cong X \otimes_{A} A$ as $A$ - $A$-bimodules. Moreover, we obtain that $\Omega_{A^{e}}^{2}\left(X \otimes_{A^{e}} D\left(A^{e}\right)\right) \cong$ $\Omega_{A^{e}}^{2}\left(X \otimes_{A} A \otimes_{A^{e}} D\left(A^{e}\right)\right) \cong X \otimes_{A} A \otimes_{A^{e}} D\left(A^{e}\right) \otimes_{A} \Omega_{A^{e}}^{2}(A)$ in $\underline{\bmod }\left(A^{e}\right)$ by 11]. Then we deduce from Lemma 2.1 that $A \otimes_{A^{e}} D\left(A^{e}\right) \otimes_{A} \Omega_{A^{e}}^{2}(A) \cong \tau_{A^{e}}(A) \operatorname{in} \underline{\bmod }\left(A^{e}\right)$. Thus $\tau_{A^{e}}(X) \cong X \otimes_{A} \tau_{A^{e}}(A)$ in $\underline{\bmod }\left(A^{e}\right)$.

We also have the following dual version of the above lemma.

Lemma 2.4. Let $A$ be a finite-dimensional, self-injective $K$-algebra. Then for every indecomposable, left-right projective $A$-A-bimodule $X$ which is not projective there is an isomorphism $\tau_{A^{e}}^{-1}(X) \cong X \otimes_{A} \tau_{A^{e}}^{-1}(A)$ in $\underline{\bmod }\left(A^{e}\right)$.

Proof. Using Lemma 2.2, repeat the arguments from the proof of Lemma 2.3.

\section{Stable equivalences of Morita type}

Two finite-dimensional $K$-algebras $A$ and $B$ are said to be stably equivalent of Morita type provided that there is an $A$-B-bimodule $N$ and a $B$ - $A$-bimodule $M$ such that the following conditions are satisfied:

(i) $M, N$ are left-right projective bimodules,

(ii) $M \otimes_{A} N \cong B \oplus \Pi$ as $B$-B-bimodules for some projective $B$ - $B$-bimodule $\Pi$,

(iii) $N \otimes_{B} M \cong A \oplus \Pi^{\prime}$ as $A$-A-bimodules for some projective $A$-A-bimodule $\Pi^{\prime}$.

Lemma 3.1. Let $A, B$ be finite-dimensional $K$-algebras. Then for every right, finite-dimensional $A \otimes_{K} B^{o p}$-module $X$ there is an isomorphism

$$
X \otimes_{A \otimes_{K} B^{o p}} D\left(A \otimes_{K} B^{o p}\right) \cong X \otimes_{A} D(A)
$$

of right $A \otimes_{K} B^{o p}$-modules.

Proof. First of all we have an isomorphism $D\left(A \otimes_{K} B^{o p}\right) \cong D(A) \otimes_{K} D\left(B^{o p}\right)$ by [12] V, Proposition 4.3]. But $D\left(B^{o p}\right) \cong B$ as $B$-B-bimodules. Then we shall indicate an isomorphism $g: X \otimes_{A \otimes_{K} B^{o p}}\left(D(A) \otimes_{K} B\right) \rightarrow B \otimes_{B} X \otimes_{A} D(A)$.

Consider a $K$-linear map $g: X \otimes_{A \otimes_{K} B^{o p}}\left(D(A) \otimes_{K} B\right) \rightarrow B \otimes_{B} X \otimes_{A} D(A)$ given by $g(x \otimes(t \otimes b))=b \otimes x \otimes t$ for any $x \in X, t \in D(A), b \in B$. Then for any $x \in X$, $t \in D(A), a \in A, b, b^{\prime} \in B$ we have $g\left([x \otimes(t \otimes b)] \cdot\left(a \otimes b^{\prime}\right)\right)=g\left(x \otimes t \cdot a \otimes b^{\prime} \cdot b\right)=$ $b^{\prime} b \otimes x \otimes t a=(b \otimes x \otimes t)\left(a \otimes b^{\prime}\right)=g(x \otimes(t \otimes b)) \cdot\left(a \otimes b^{\prime}\right)$. Thus $g$ is a homomorphism of right $A \otimes_{K} B^{o p}$-modules.

For any $b \in B, x \in X, t \in D(A)$ consider the element $b \otimes x \otimes t \in B \otimes_{B} X \otimes_{A} D(A)$. Then $b \otimes x \otimes t=g(x \otimes(t \otimes b))$. Thus $g$ is an epimorphism. 
The same reasoning shows that $f: B \otimes_{B} X \otimes_{A} D(A) \rightarrow X \otimes_{A \otimes_{K} B^{o p}}\left(D(A) \otimes_{K} B\right)$ given by the formula $f(b \otimes x \otimes t)=x \otimes(t \otimes b), x \in X, t \in D(A), b \in B$, is a homomorphism of right $A \otimes_{K} B^{o p}$-modules. Furthermore, it is clear that $f g=$ id, $g f=\mathrm{id}$. Consequently, $g$ is an isomorphism. Since $B \otimes_{B} X \otimes_{A} D(A) \cong X \otimes_{A} D(A)$, the lemma is proved.

Lemma 3.2. Let $A, B$ be finite-dimensional $K$-algebras. Thenfor every left, finitedimensional $B \otimes_{K} A^{\text {op }}$-module $X$ there is an isomorphism $D\left(B \otimes_{K} A^{o p}\right) \otimes_{B} \otimes_{K} A^{\text {op }}$ $X \cong D(B) \otimes_{B} X$ of left $B \otimes_{K} A^{o p}$-modules.

Proof. Arguments similar to those used in the proof of Lemma 3.1 show the lemma.

Corollary 3.3. Let $A, B$ be finite-dimensional, self-injective $K$-algebras. Then for every indecomposable, left-right projective $B$-A-module $X$ it holds that $\tau_{A \otimes_{K} B^{o p}}(X)$ $\cong X \otimes_{A} \tau_{A^{e}}(A) \cong \tau_{B^{e}}(B) \otimes_{B} X$ in $\underline{\bmod }\left(A \otimes_{K} B^{o p}\right)$.

Proof. We infer by the formula $\tau_{A \otimes_{K} B^{o p}} \cong \Omega_{A \otimes_{K} B^{o p}} \circ\left(-\otimes_{A \otimes_{K} B^{o p}} D\left(A \otimes_{K} B^{o p}\right)\right)$ that $\tau_{A \otimes_{K} B^{o p}}(X) \cong \Omega_{A \otimes_{K} B^{o p}}\left(X \otimes_{A \otimes_{K} B^{o p}} D\left(A \otimes_{K} B^{o p}\right)\right)$. Then we deduce from Lemma 3.1 that $X \otimes_{A \otimes_{K} B^{o p}} D\left(A \otimes_{K} B^{o p}\right) \cong X \otimes_{A} D(A)$. Thus $\tau_{A \otimes_{K} B^{o p}}(X) \cong$ $\Omega_{A \otimes_{K} B^{o p}}\left(X \otimes_{A} D(A)\right)$. Using [11] we have $\Omega_{A \otimes_{K} B^{o p}}^{2}\left(X \otimes_{A} D(A)\right) \cong X \otimes_{A} D(A) \otimes_{A}$ $\Omega_{A^{e}}^{2}(A)$. Since $X \otimes_{A} D(A) \cong A \otimes_{A} X \otimes_{A} D(A)$, we conclude by Lemma 3.1 that $X \otimes_{A} D(A) \cong X \otimes_{A^{e}} D\left(A^{e}\right)$. Combining all the above isomorphisms we get that $\tau_{A \otimes_{K} B^{o p}}(X) \cong X \otimes_{A} \tau_{A^{e}}(A)$ in $\underline{\bmod }\left(A \otimes_{K} B^{o p}\right)$.

Since $X$ is also a left $B \otimes_{K} A^{o p}$-module and $\tau_{B \otimes_{K} A^{o p}}(X) \cong \tau_{A \otimes_{K} B^{o p}}(X)$ then in view of Lemma 3.2 we can repeat all the above arguments and get that $\tau_{B \otimes_{K} A^{o p}}(X)$ $\cong \tau_{B^{e}}(B) \otimes_{B} X$ in $\underline{\bmod }\left(A \otimes_{K} B^{o p}\right)$. This finishes our proof.

Lemma 3.4. Let $A$ and $B$ be finite-dimensional, self-injective $K$-algebras which are stably equivalent of Morita type. Let ${ }_{B} M_{A}$ and ${ }_{A} N_{B}$ be bimodules which establish this equivalence between $A$ and $B$. Then for any non-negative integer $n$ there is an isomorphism $M \otimes_{A} \tau_{A^{e}}^{n}(A) \otimes_{A} N \cong \tau_{B^{e}}^{n}(B)$ in $\underline{\bmod }\left(B^{e}\right)$.

Proof. We shall show our lemma inductively on $n$. First observe that for $n=0$ we have $M \otimes_{A} A \otimes_{A} N \cong M \otimes_{A} N \cong B \oplus \Pi$, where $\Pi$ is a right projective $B^{e}$-module. Thus $M \otimes_{A} A \otimes_{A} N \cong B$ in $\underline{\bmod }\left(B^{e}\right)$.

Now we assume that for some non-negative integer $n$ there is an isomorphism $M \otimes_{A} \tau_{A^{e}}^{n}(A) \otimes_{A} N \cong \tau_{B^{e}}^{n}(B)$ in $\underline{\bmod }\left(B^{e}\right)$. Then we infer by Corollary 3.3 that $\tau_{B^{e}}\left(\tau_{B^{e}}^{n}(B)\right) \cong \tau_{B^{e}}\left(M \otimes_{A} \tau_{A^{e}}^{n}(A) \otimes_{A} N\right) \cong M \otimes_{A} \tau_{A^{e}}^{n}(A) \otimes_{A} N \otimes_{B} \tau_{B^{e}}(B)$. But again applying Corollary 3.3 to $N \otimes_{B} \tau_{B^{e}}(B)$ we obtain that $N \otimes_{B} \tau_{B^{e}}(B) \cong \tau_{A^{e}}(A) \otimes_{A} N$ in $\underline{\bmod }\left(B \otimes_{K} A^{o p}\right)$. This means that there is a right projective $B \otimes_{K} A^{o p}$-module $P$ such that $N \otimes_{B} \tau_{B^{e}}(B) \cong \tau_{A^{e}}(A) \otimes_{A} N \oplus P$. Therefore we have $M \otimes_{A} \tau_{A^{e}}^{n}(A) \otimes_{A}$ $N \otimes_{B} \tau_{B^{e}}(B) \cong M \otimes_{A} \tau_{A^{e}}^{n}(A) \otimes_{A} \tau_{A^{e}}(A) \otimes_{A} N \oplus M \otimes_{A} \tau_{A^{e}}^{n}(A) \otimes_{A} P$. Applying [14, Lemma 1.6] we know that $M \otimes_{A} \tau_{A^{e}}^{n}(A) \otimes_{A} P$ is a projective right $B^{e}$-module. Furthermore, we deduce from Corollary 3.3 that $\tau_{A^{e}}^{n}(A) \otimes_{A} \tau_{A^{e}}(A) \cong \tau_{A^{e}}^{n+1}(A)$ in $\underline{\bmod }\left(A^{e}\right)$. Thus $M \otimes_{A} \tau_{A^{e}}^{n}(A) \otimes_{A} \tau_{A^{e}}(A) \otimes_{A} N \cong M \otimes_{A} \tau_{A^{e}}^{n+1}(A) \otimes_{A} N$. Consequently, $\tau_{B^{e}}^{n+1}(B) \cong \tau_{B^{e}}\left(\tau_{B^{e}}^{n}(B)\right) \cong M \otimes_{A} \tau_{A^{e}}^{n+1}(A) \otimes_{A} N$ in $\underline{\bmod }\left(B^{e}\right)$ and the lemma follows.

Lemma 3.5. Let $A$ and $B$ be finite-dimensional, self-injective $K$-algebras which are stably equivalent of Morita type. Let ${ }_{B} M_{A}$ and ${ }_{A} N_{B}$ be bimodules which establish this equivalence between $A$ and $B$. Then for every non-negative integer $n$ there is an isomorphism $N \otimes_{B} M \otimes_{A} \tau_{A^{e}}^{n}(A) \otimes_{A} N \otimes_{B} M \cong \tau_{A^{e}}^{n}(A)$ in $\underline{\bmod }\left(A^{e}\right)$.

Proof. Apply Lemma 3.4 twice. 
Given a self-injective, finite-dimensional $K$-algebra $C$, it is well-known that its enveloping algebra $C^{e}$ is also self-injective. Consider the full subcategory in $\underline{\bmod }\left(C^{e}\right)$ which is formed by the finite direct sums of objects isomorphic to $\tau_{C^{e}}^{n}(C)$ for non-negative integers $n$. Denote this subcategory by $\underline{\bmod }_{C}^{\tau}\left(C^{e}\right)$. It plays a crucial role in our proof of the main results.

For two finite-dimensional self-injective $K$-algebras $A$ and $B$, assume they are stably equivalent of Morita type. Suppose that the bimodules ${ }_{B} M_{A}$ and ${ }_{A} N_{B}$ yield their stable equivalence. Now our goal is to show that the functor $M \otimes_{A}-\otimes_{A}$ $N: \bmod \left(A^{e}\right) \rightarrow \bmod \left(B^{e}\right)$ induces an equivalence of the categories $\underline{\bmod }_{A}^{\tau}\left(A^{e}\right)$ and $\underline{\bmod }_{B}^{\tau}\left(B^{e}\right)$

Proposition 3.6. There exists an equivalence $F: \underline{\bmod }_{A}^{\tau}\left(A^{e}\right) \rightarrow \underline{\bmod }_{B}^{\tau}\left(B^{e}\right)$ such that for every non-negative integer $n$ it holds that $F\left(\tau_{A^{e}}^{n}(A)\right) \cong \tau_{B^{e}}^{n}(B)$ in $\underline{\bmod }_{B}^{\tau}\left(B^{e}\right)$.

Proof. In order to prove the proposition we have to define a functor $F: \underline{\bmod }_{A}^{\tau}\left(A^{e}\right) \rightarrow$ $\underline{\bmod }_{B}^{\tau}\left(B^{e}\right)$. For every object $X$ of $\underline{\bmod }_{A}^{\tau}\left(A^{e}\right)$ we put $F(X)=M \otimes_{A} X \otimes_{A} N$. For every morphism $\underline{f}: X \rightarrow Y$ of $\underline{\bmod }_{A}^{\tau}\left(A^{e}\right)$ we put $F(\underline{f})=\underline{1_{M} \otimes f \otimes 1_{N}}$. A handy verification shows that $F$ is well-defined.

Now we can similarly define a functor $G: \underline{\bmod }_{B}^{\tau}\left(B^{e}\right) \rightarrow \underline{\bmod }_{A}^{\tau}\left(A^{e}\right)$. We put $G(U)=N \otimes_{B} U \otimes_{B} M$ for every object $U$ of $\underline{\bmod }_{B}^{\tau}\left(B^{e}\right)$. For every morphism $\underline{g}: U \rightarrow V$ of $\underline{\bmod }_{B}^{\tau}\left(B^{e}\right)$ we put $F(\underline{g})=\underline{1_{N} \otimes g \otimes 1_{M}}$.

We infer by Lemma 3.5 that for every object $X$ of $\underline{\bmod }_{A}^{\tau}\left(A^{e}\right)$ we have $G F(X) \cong$ $X$ in $\bmod _{A}^{\tau}\left(A^{e}\right)$. Thus the composed functor $G F$ is dense. Now consider a morphism $f: X \rightarrow Y$ of $\underline{\bmod }_{A}^{\tau}\left(A^{e}\right)$. Then $G F(f)=G\left(1_{M} \otimes f \otimes 1_{N}\right)=$ $\underline{1_{N} \otimes 1_{M} \otimes f \otimes 1_{N} \otimes 1_{M}}=1_{A \oplus \Pi^{\prime}} \otimes f \otimes 1_{A \oplus \Pi^{\prime}}=1_{A} \otimes f \otimes 1_{A}=f$. Therefore the composed functor $G F$ is fully faithful. Hence $F$ is an equivalence of categories.

Proof of Theorem 1.1. Let $A$ and $B$ be self-injective, finite-dimensional $K$-algebras which are stably equivalent of Morita type. Then we infer by Proposition 3.6 that $\mathbb{A}\left(\tau_{A^{e}} ; A\right) \cong \mathbb{A}\left(\tau_{B^{e}} ; B\right)$ as $K$-linear spaces.

In order to finish our proof we need to show the following fact. For any morphism $\underline{g}: \tau_{A^{e}}^{n}(A) \rightarrow \tau_{A^{e}}^{m}(A)$ it holds that $\underline{1_{M} \otimes \tau_{A^{e}}(g) \otimes 1_{N}}=\tau_{B^{e}}\left(\underline{1_{M} \otimes g \otimes 1_{N}}\right)$, where $n, m$ are some non-negative integers and $\tau_{A^{e}}(g)$ stands for a representative of the coset $\tau_{A^{e}}(\underline{g})$.

To prove this fact we shall use the natural isomorphism $\tau_{C} \cong \Omega_{C}^{2} \circ\left(-\otimes_{C} D(C)\right)$ of functors. Then we have

$$
\left.\left.\tau_{B^{e}} \underline{\left(\underline{1_{M} \otimes g \otimes 1_{N}}\right)}=\Omega_{B^{e}}^{2} \underline{\left(1_{M} \otimes g \otimes 1_{N} \otimes 1_{D\left(B^{e}\right)}\right.}\right)=\Omega_{B^{e}}^{2} \underline{\left(1_{M} \otimes g \otimes 1_{D\left(A^{e}\right)} \otimes 1_{N}\right.}\right) .
$$

Now we consider the commutative diagram

$$
\begin{aligned}
& 0 \rightarrow \Omega_{A^{e}}\left(\tau_{A^{e}}^{n}(A) \otimes_{A^{e}} D\left(A^{e}\right)\right) \quad \rightarrow \quad P \quad \rightarrow \quad \tau_{A^{e}}^{n}(A) \otimes_{A^{e}} D\left(A^{e}\right) \rightarrow 0 \\
& \downarrow \Omega_{A^{e}}\left(g \otimes 1_{D\left(A^{e}\right)}\right) \quad \downarrow h \quad \downarrow g \otimes 1_{D\left(A^{e}\right)} \\
& 0 \rightarrow \Omega_{A^{e}}\left(\tau_{A^{e}}^{m}(A) \otimes_{A^{e}} D\left(A^{e}\right)\right) \rightarrow Q \quad \rightarrow \quad \tau_{A^{e}}^{m}(A) \otimes_{A^{e}} D\left(A^{e}\right) \rightarrow 0
\end{aligned}
$$

whose rows are exact, where $P \rightarrow \tau_{A^{e}}^{n}(A) \otimes_{A^{e}} D\left(A^{e}\right), Q \rightarrow \tau_{A^{e}}^{m}(A) \otimes_{A^{e}} D\left(A^{e}\right)$ are minimal projective covers in $\bmod \left(A^{e}\right)$. Hence we obtain the following commutative 
diagram in $\bmod \left(B^{e}\right)$ :

$$
\begin{aligned}
& 0 \rightarrow M \otimes_{A} \Omega_{A^{e}}\left(\tau_{A^{e}}^{n}(A) \otimes_{A^{e}} D\left(A^{e}\right)\right) \otimes_{A} N \rightarrow M \otimes_{A} P \otimes_{\tilde{h}} N \\
& \downarrow \tilde{f} \quad \downarrow \tilde{h} \\
& 0 \rightarrow \quad M \otimes_{A} \Omega_{A^{e}}\left(\tau_{A^{e}}^{m}(A) \otimes_{A^{e}} D\left(A^{e}\right)\right) \otimes_{A} N \rightarrow M \otimes_{A} Q \otimes_{A} N \\
& \rightarrow \quad M \otimes_{A} \tau_{A^{e}}^{n}(A) \otimes_{A^{e}} D\left(A^{e}\right) \otimes_{A} N \quad \rightarrow 0 \\
& \downarrow \tilde{g} \\
& \rightarrow \quad M \otimes_{A} \tau_{A^{e}}^{m}(A) \otimes_{A^{e}} D\left(A^{e}\right) \otimes_{A} N \quad \rightarrow 0
\end{aligned}
$$

whose rows are exact, where $\tilde{f}=1_{M} \otimes \Omega_{A^{e}}\left(g \otimes 1_{D\left(A^{e}\right)}\right) \otimes 1_{N}, \tilde{h}=1_{M} \otimes h \otimes 1_{N}, \tilde{g}=$ $1_{M} \otimes g \otimes 1_{D\left(A^{e}\right)} \otimes 1_{N}$. Since $M \otimes_{A} P \otimes_{A} N, M \otimes_{A} Q \otimes_{A} N$ are projective $B^{e}$-modules by [14], we have $\Omega_{B^{e}}\left(1_{M} \otimes g \otimes 1_{D\left(A^{e}\right)} \otimes 1_{N}\right)=1_{M} \otimes \Omega_{A^{e}}\left(g \otimes 1_{D\left(A^{e}\right)}\right) \otimes 1_{N}$.

The same reasoning shows that

$$
\Omega_{B^{e}}^{2} \underline{\left(1_{M} \otimes g \otimes 1_{D\left(A^{e}\right)} \otimes 1_{N}\right)}=\underline{1_{M} \otimes \Omega_{A^{e}}^{2}\left(g \otimes 1_{D\left(A^{e}\right)}\right) \otimes 1_{N}} .
$$

Therefore we get the equality

$$
\tau_{B^{e}}\left(\underline{1_{M} \otimes g \otimes 1_{N}}\right)=\underline{1_{M} \otimes \tau_{A^{e}}(g) \otimes 1_{N}},
$$

which shows the above fact.

Using the proved fact and Proposition 3.6 we obtain that for any morphisms $\underline{g}: \tau_{A^{e}}^{n}(A) \rightarrow A$ and $\underline{h}: \tau_{A^{e}}^{m}(A) \rightarrow A$ it holds that $F\left(\underline{g} \circ \tau_{A^{e}}^{n}(\underline{h})\right)=F(\underline{g}) \circ \tau_{B^{e}}^{n}(F(\underline{h}))$, where $F: \underline{\bmod }_{A}^{\tau}\left(A^{e}\right) \rightarrow \underline{\bmod }_{B}^{\tau}\left(B^{e}\right)$ is the equivalence induced by the functor $M \otimes_{A}$ $-\otimes_{A} N: \overline{\bmod }\left(A^{e}\right) \rightarrow \bmod \left(B^{e}\right)$. Thus the $K$-linear isomorphism of $\mathbb{A}\left(\tau_{A^{e}} ; A\right)$ and $\mathbb{A}\left(\tau_{B^{e}} ; B\right)$ is an isomorphism of $K$-algebras and our proof is finished.

\section{Derived EQUivalenCES}

Let $C$ be a finite-dimensional $K$-algebra. One can attach a category $\mathbf{D}^{b}(\operatorname{Mod}(C))$ to the category of all right $C$-modules. The category $\mathbf{D}^{b}(\operatorname{Mod}(C))$ is the derived category of all bounded complexes of right $C$-modules. It is a well-known triangulated category which was studied by several authors (see [1, 2], 6], [15]). Two $K$-algebras $A$ and $B$ are said to be derived equivalent if their derived categories $\mathbf{D}^{b}(\operatorname{Mod}(A))$ and $\mathbf{D}^{b}(\operatorname{Mod}(B))$ are equivalent as triangulated categories.

Proof of Theorem 1.2. Suppose that $A, B$ are finite-dimensional, self-injective $K$ algebras which are derived equivalent. Then we infer by [15, Corollary 5.5] that $A$ and $B$ are stably equivalent of Morita type. Thus we deduce by Theorem 1.1 that the $K$-algebras $\mathbb{A}\left(\tau_{A^{e}} ; A\right)$ and $\mathbb{A}\left(\tau_{B^{e}} ; B\right)$ are isomorphic.

\section{REFERENCES}

1. H. Asashiba, A covering technique for derived equivalence, J. Algebra 191(1997), 382-415. MR 98b:16009

2. H. Asashiba, The derived equivalence classification of representation-finite selfinjective algebras, J. Algebra 214(1999), 182-221. MR 2000g:16019

3. M. Auslander, I. Reiten and S. Smalo, Representation Theory of Artin Algebras, Cambridge Studies in Advanced Math. Vol. 36, Cambridge Univ. Press (Cambridge, 1995). MR 96e:16015

4. M. Broué, Equivalences of Blocks of Group Algebras, in: V. Dlab and L.L. Scott (eds.) Finite Dimensional Algebras and Related Topics, NATO ASI Series C Vol. 424, Kluwer Academic Press (Dodrecht, 1992), 1-26. MR 97c:20004

5. P. Gabriel, Auslander-Reiten sequences and representation-finite algebras, Lecture Notes in Math. 831(Springer-Verlag, Berlin, 1980), 1-71. MR 82i:16030

6. D. Happel, Triangulated categories in the representation theory of finite-dimensional algebras, London Math. Soc. Lecture Notes 119, Cambridge Univ. Press (Cambridge, 1988). MR 89e:16035 
7. D. Happel, Hochschild cohomology of finite-dimensional algebras, in: Seminair d'Algebre P. Dubriel et M-P.Maliavin, Lecture Notes in Math. 1404(Springer-Verlag, Berlin, 1989), 108126. MR 91b:16012

8. A. Heller, The loop-space functor in homological algebra, Trans. Amer. Math. Soc. 96(1960), 382-394. MR 22:6840

9. O. Kerner, Minimal approximations, orbital elementary modules, and orbit algebras of regular modules, J. Algebra 217(1999), 528-554. MR 2000e:16018

10. H. Lenzing, Wild Canonical Alebras and Rings of Automorphic Forms, in: V. Dlab and L.L. Scott (eds.) Finite Dimensional Algebras and Related Topics, NATO ASI Series C Vol. 424, Kluwer Academic Press (Dodrecht, 1992), 191-212. MR 95m:16008

11. M. Linckelmann, Stable equivalences of Morita type for self-injective algebras and p-groups, Math. Z. 223(1996), 87-100. MR 97j:20011

12. S. Mac Lane, Homology, (Springer-Verlag, Berlin, 1963). MR 28:122

13. Z. Pogorzały, Invariance of Hochschild cohomology algebras under stable equivalences of Morita type, J. Math. Soc. Japan 53(2001), 913-918.

14. Z. Pogorzały, Left-right projective bimodules and stable equivalences of Morita type, Colloq. Math. Vol.88 (2) (2001), 243-255.

15. J. Rickard, Derived equivalences as derived functors, J. London Math. Soc. (2) 43(1991), 37-48. MR 92b:16043

Faculty of Mathematics and Computer Science, Nicholas Copernicus University, Chopina 12/18, 87-100 Toruń, Poland

E-mail address: zypo@mat.uni.torun.pl 